\title{
Géneros, generaciones y lugares: cambios en el medio rural de Chile Central
}

\author{
Ximena Valdés S. \\ CEDEM, Santiago, Chile. \\ Email: ximenavaldess@gmail.com \\ Loreto Rebolledo G. \\ Universidad de Chile, Santiago, Chile. \\ Email:mareboll@u.uchile.cl
}

\begin{abstract}
Resumen: ${ }^{1}$ Este artículo da cuenta de hallazgos de una investigación FONDECYT ( $\left.\mathrm{N}^{\circ} 1120425\right)$ que se propuso conocer cómo hombres y mujeres han experimentado y han sido parte de los cambios estructurales, sociales y culturales ocurridos en diferentes lugares de Chile Central a partir de la segunda mitad del siglo XX hasta la actualidad. Para ello se ha privilegiado técnicas cualitativas como entrevistas en profundidad a hombres y mujeres de distintas edades y la aplicación del método genealógico a tres generaciones en dos grupos de parentesco residentes en áreas de riego y secano.
\end{abstract}

Palabras clave: cambio social, lugares, géneros y generaciones

\section{Gender, generations and places: changes in rural areas of Central Chile}

\begin{abstract}
This paper presents discoveries of a FONDECYT ( $\left.\mathrm{N}^{\circ} 1120425\right)$ investigation that intended to know how men and women have experienced and how they have been part of the structural, social and cultural changes occurred in different places of Central Chile in the last fifty years, giving priority to qualitative methods as interviews to men and women of different ages and the use of the genealogical method for three generations in two groups of relationship residents in irrigated and unirrigated land.
\end{abstract}

Keywords: social change; places; gender; generations

\section{Gêneros, gerações e lugares: mudanças nas áreas rurais do Chile Central}

Resumo: Este artigo relata os resultados de uma pesquisa FONDECYT (No. 1120425), que é a de saber como os homens e mulheres têm experimentado e sido parte das mudanças estruturais, sociais e culturais acontecidos em diferentes partes do Chile Central desde a segunda metade do século XX até o presente. Para isso temos privilegiado técnicas qualitativas tais como entrevistas em profundidade com homens e mulheres de diferentes idades e a aplicação do método genealógico a 
três gerações em dois grupos de parentesco residentes em áreas irrigadas e sem irrigação.

Palavras-chave: mudança social, lugares, gêneros e gerações.

\section{Introducción}

La reforma agraria rompió con las relaciones sociales de dominación de larga duración que existían en el medio rural aun cuando el régimen de hacienda y el sistema de inquilinaje (Bauer, 1994; Bengoa, 1988) coexistieron con procesos de modernización temprana que produjeron diferencias territoriales y entre distintos tipo de explotaciones agrícolas (Santana, 2006). La redistribución de tierras, el acceso de los campesinos a recursos crediticios y maquinaria agrícola, la explosión del sindicalismo agrario (Affonso et. al., 1970 ), la expansión de la institucionalidad pública de la ciudad al campo, la conquista de derechos laborales (Tinsman, 2009) vinieron acompañados de una profunda reforma educacional que incorporó a los hijos e hijas de campesinos y trabajadores agrícolas a la extensión de los años de escolaridad introduciéndose por este hecho modificaciones en las forma de vida en el medio rural (Osandón et al., 2014). Con la expansión de las escuelas al campo y el aumento de los años de estudio de niñas y niños se rompía con la tutela exclusiva que tenían los padres sobre sus hijos. Se produjo una paulatina retracción de la infancia del trabajo en la agricultura, nuevos referentes para niños y jóvenes de ambos sexos y una sociabilidad que abrió las relaciones más allá de la familia y el parentesco. En segundo lugar durante este período se inauguró un proceso tendiente al logro de las mujeres de la soberanía sobre sus cuerpos con las políticas de control de natalidad. Pese a que fueron frenadas bajo la dictadura militar desencadenaron un proceso ininterrumpido de disminución del número de hijos por mujer, de promedios de 5,58 hijos por mujer en 1960, 5 hijos en 1965, 2,68 en 1980, 2,09 el año 2000 y 1,9 el 2004. Estos dos últimos aspectos, aumento de la escolaridad y control de la natalidad se mantuvieron como legado del período de reforma agraria y del proceso de modernización que la sociedad chilena emprendió en los años sesenta.

La contrarreforma agraria privatizó la tierra expropiada al latifundio limitando los derechos sociales y laborales conquistados por campesinos y trabajadores agrícolas entre los años 1964-1973. Bajo el modelo neoliberal el mercado reconfiguró el espacio rural, reordenó la estructura agraria, las relaciones laborales y las formas de poblamiento (Canales y Canales, 2013; Bengoa, 2013). Las políticas silvo-agropecuarias y de comercio exterior promovieron la expansión de las empresas exportadoras mientras el Estado congeló sus políticas de promoción campesina y el mercado se transformó en el eje articulador del desarrollo agrario y rural bajo un dinamismo empresarial-exportador que contribuyó a la concentración de la propiedad de la tierra (Gómez, 2014). 
Desde los años ochenta en adelante el espacio rural se transformó en un nuevo espacio de inversiones nacionales y extranjeras lo que contribuyó a reconfigurar el territorio (Harvey, 2007) a partir de la reconversión de la agricultura al modelo exportador. En las últimas décadas ha habido procesos de fragmentación y diferenciación social en el campo, fenómenos de desagrarización como los estudiados en México (De Grammont, 2009), causados por la disminución de las pequeñas explotaciones, de los campesinos pequeño productores junto al aumento de hogares rurales sin tierras. Ello ha ido de la mano de la diversificación de los ingresos campesinos, el aumento de los empleos no rurales (Kay, 2009; Dirven, 2004) y una gran expansión de la asalarización de la población rural junto a su feminización (Valdés, 1988) y la inclusión de asalariados provenientes de ciudades en las faenas agrícolas (Caro, 2012), de migraciones de los países andinos y de migraciones internas de campesinos e indígenas, constituyéndose una suerte de nomadismo asalariado en cada temporada a través de territorios migratorios creados a partir de la demanda de trabajadores de la agricultura de exportación (Valdés, 2012). La mayoría son empleos agrícolas temporales y una parte significativa son empleos precarios e informales.

La democratización política a partir de 1990 no modificó la estructura agraria ni introdujo cambios sustantivos en lo referente a las relaciones laborales, formas de contratación y sindicalización de trabajadores agrícolas; sin embargo, se diseñaron un conjunto de políticas públicas para mitigar la abultada pobreza y mejorar las formas de vida de la población rural: ampliación de infraestructura, red de caminos y carreteras, electrificación, acceso al agua potable, electrificación. subsidio a la vivienda , programas de promoción a la producción para pequeños propietarios, hombres y mujeres así como políticas de promoción de la mujer. Por su parte, el mercado de bienes y servicios dio acceso a artefactos domésticos, TV y telefonía celular a través de créditos de consumo acercando a la población del campo a la ciudad y de esta manera contribuyendo al desdibujamiento las fronteras campo-ciudad. El celular, el computador y la mejor conectividad van a facilitar contactos y acceso a la información entre los jóvenes que, a diferencia de sus abuelos, han ampliado sus años de escolaridad.

Tales fenómenos surgen bajo los nuevos escenarios creados por el posfordismo en el medio rural en actividades agro-exportadoras como las forestales, agroindustriales, vitivinícolas, frutícolas y hortícolas (Valdés et al, 2014).

En el marco de estos procesos desencadenados a partir de la reforma agraria la mayor parte de las investigaciones han analizado temas y problemas como los cambios en la estructura agraria producto de la contra-reforma (Ortega, 1987; Gómez y Echenique, 2009), en el uso del suelo y en la participación de distinto tipo de explotaciones en la producción (Bengoa, 2013), en las formas de poblamiento (Canales et. al., op. cit); la patrimonialización territorial asociada a ciertas orientaciones productivas (Bengoa, 2012), la emergencia de nuevos actores e instituciones en el campo (PNUD, 2008), entre otros. Sin embargo, en los estudios de sociología, 
geografía y antropología rural no ha habido un preocupación especial sobre la manera en que hombres y mujeres han vivido estas transformaciones y las consecuencias sobre ellos de la superposición de un modelo de desarrollo redistributivo como la reforma agraria seguido de un modelo neoliberal que ha internacionalizado la agricultura y extendido la flexibilización laboral.

Este artículo se basa en una investigación que buscó comprender cómo inciden las transformaciones estructurales en sujetos de distinto sexo y edad estudiando los cambios intergeneracionales en cuatro localidades rurales. Se buscaba comprender la incidencia de la residencia y del lugar en cambios en la familia, el trabajo y las identidades de género a partir de cuatro estudios de caso (de los cuales dos son analizados en este artículo) en localidades en zonas de riego y secano. Este objetivo, dada la profunda diferenciación espacio-territorial que se ha producido en el país con el modelo agro-exportador, busca aportar desde el análisis micro-social a la comprensión de las transformaciones intergeneracionales.

Valiéndonos de la flexibilidad que caracteriza a los estudios cualitativos (Tarrés 2004: 21), en el proceso de investigación abordamos los procesos de cambios dando cuenta tanto de los contextos en que estos se produjeron y los efectos objetivos y subjetivos en los hombres y mujeres que los vivieron. Para ello se utilizó información obtenida de fuentes secundarias y, de documentos escritos, estadísticas, así como de relatos biográficos.

Después de más de tres décadas de trabajo en zonas rurales de Chile Central con hombres y mujeres dando cuenta de sus actividades productivas, relaciones familiares, nuevas inserciones laborales, etc., tanto en las historias de vida como en las entrevistas en profundidad realizadas aparecían ciertas recurrencias (modos de hacer, de funcionar y operativizar los recursos familiares para lograr cambios económicos y sociales) que traspasaban las familias y las localidades y que eran pesquisables en más de una generación.

Como una forma de corroborar las hipótesis levantadas a partir de estas recurrencias se decidió hacer estudios de caso en localidades diferentes abarcando tres generaciones. Para su selección se usó como criterio la pertenencia a localidades del valle central que han tenido diferentes desarrollos productivos a partir de la década de los 80, y donde en el pasado coexistieron fundos con pequeñas propiedades.

Los estudios de caso fueron utilizados para realizar comprobaciones (Guba y Lincoln, 1981) buscando profundizar la comprensión de situaciones empíricas (Gunderman, 2004) a partir de casos múltiples, de modo de poder comparar y establecer semejanzas y diferencias. Estos estudios de caso fueron alimentados por las entrevistas e historias de vida hechas en distintos períodos entre y 1981 y $2014^{2}$ en las cuatro localidades y por las notas de campo obtenidas de la observación. 
El criterio de selección de los grupos familiares se tomó en consideración el que fueran representativos de dos tipos de situaciones, descendientes de inquilinos y de pequeños propietarios. Una vez que se revisó el material recolectado en periodos anteriores, entre 2012 y 2014 se completó los vacíos de información respecto a algunos integrantes de los grupos familiares escogidos y a partir de sucesivas entrevistas a diferentes personas de la familia, por rama materna y paterna y se comenzó a trabajar con las genealogías ${ }^{3}$ completando información de ascendientes y descendientes.

A través de la construcción de mapas de parentesco - anexos-(Harris, 1986: 19; Héritier, 1987) se rescató por medio de entrevistas en profundidad las trayectorias sociales de hombres y mujeres (aspectos residenciales, de alianza y filiación, laborales, migratorios, nivel de estudios, etc.). Esto permitió conocer los cambios, similitudes y diferencias intergeneracionales, entre lugares, las subjetividades colectivas (Domingues, 2002), los elementos que se transmiten entre géneros y generaciones así como las rupturas y reproducción de factores ligados a la posición social o a la diferencia sexual (Davinson, 2006; Montecino et al., 1993; Lara, 2010).

El trabajo genealógico fue central para comprender el peso de la historia sobre los destinos individuales y capturar aspectos que diferencian a las generaciones (Lara, 2010 ). En el plano sociológico permite identificar aspectos demográficos, económicos, profesionales, culturales y geográficos de las estructuras familiares. A partir de las posiciones sociales y de status de sus miembros a lo largo de varias generaciones se ponen en evidencia la o las clases de pertenencia, los fenómenos de ascenso o de regresión, las estrategias de alianza y las rupturas matrimoniales; la movilidad social, retroceso de la mortalidad infantil y reducción de la natalidad, entre otros (De Gaulejac, 1999). El enfoque de género (Lamas, 1913; Héritier, 1996) complementa la mirada sobre los cambios socio-culturales entendiendo que hombres y mujeres no los experimentan de la misma forma y que la fuerza social de la inercia mantiene no obstante algunos cambios las asimetrías sexuales (Testart 2014).

Este artículo da cuenta de los cambios en las identidades de género de hombres y mujeres de tres generaciones de dos grupos familiares que han sido protagonistas de los procesos de cambio en el agro, para lo cual nos centraremos en los resultados de dos de los estudios de caso, específicamente, de los inquilinos de fundo: Lo Ermita en un área de riego y Pilén en el secano costero.

La aplicación de las técnicas cualitativas tiene como punto de partida la generación nacida entre las décadas de 1920 a 1940 (generación mayor), hijos e hijas (generación intermedia) y los nietos/as (generación joven). El material recogido en entrevistas en profundidad, entrevistas grupales y observación participante en dos localidades corresponde a dos familias que dieron origen a hijos/as y nietos/as a las que se aplicó el método genealógico incluyendo en las entrevistas a los/as miembros/as que se incorporaron por alianza a ambos grupos de parentesco. 
El corpus del material empírico lo constituyen diez entrevistas en profundidad a las dos parejas de la primera generación en los fundos Lo Ermita y Aldea de Pilén (en distintos años y más de diez entrevistas a personas de la misma edad, lugar y condición social en estas localidades); veinte entrevistas a la generación intermedia -hijos/as, yernos y nueras- y diez entrevistas a la generación joven. Ambas parejas vivían en los años ochenta en los fundos Lo Ermita de Calera de Tango y el fundo La Aldea de Pilén en Cauquenes. La familia que se originó en el fundo Lo Ermita y algunos de sus hijos y nietos/as todavía viven en el fundo a diferencia de la familia que trabajaba en esos años en el fundo La Aldea que viven fuera del fundo, en la localidad de Pilén, área de pequeña propiedad de hábitat disperso.

Para contextualizar los estudios de caso y conocer las transformaciones en la agricultura se analizó los cambios en el uso del suelo y en el poblamiento revisando planos aerofotogramétricos y fotografías satelitales de diferentes años abarcando las décadas del setenta, noventa y dos mil y Censos Agrícolas de 1997 y 2007.

\section{Lugares, paisajes y poblaciones}

Las políticas neoliberales aplicadas a la agricultura modificaron el paisaje, la orientación productiva de los territorios, las formas de asentamiento de la población, los lugares, el empleo, las formas de vida. Volcaron una parte sustantiva de la producción agrícola al mercado externo marcando el carácter exportador de algunos territorios dando curso a la apertura de nuevos espacios para el capital (Harvey, 2007). El contexto territorial en que se localizan los casos estudiados se inscribe en dos tipos de procesos: la urbanización (Calera de Tango, Lo Ermita) y la expansión de la silvicultura (Cauquenes, Pilén).

La comuna de Calera de Tango se ubica en el Valle del Maipo. Los centros poblados cercanos como Santiago y sus alrededores dan cuenta de la urbanización de comunas que han sido sometidas a un fuerte proceso de subdivisión de la tierra y de conurbanización con la ciudad marcado además por la expansión de zonas de vivienda social cuyo acceso es posible por los subsidios públicos ${ }^{4}$ a lo que se suma el crecimiento de parcelas de agrado de propiedad de citadinos de sectores acomodados. Esto explica la disminución de 4006 propiedades agrícolas (de 16.803 a 12.797) y de 110 mil hectáreas destinadas a la agricultura en la Región Metropolitana entre los Censos Agropecuarios de 1997 y 2007.

El Maipo es un valle de tierras fértiles concentradas hasta comienzos del siglo $\mathrm{XX}$ en grandes propiedades. Un proceso de subdivisión ${ }^{5}$ muy temprano se dio paralelamente al cambio en el uso del suelo. En 1928 las grandes propiedades se dedicaban a los cultivos extensivos de cereales y pastoreo (Martin, 1960) y casi todas las casas patronales de los fundos estaban rodeadas de viñas. El proceso de subdivisión posterior condujo a que la mayoría de las propiedades agrícolas medianas y pequeñas, se dedi- 
caran casi exclusivamente a las viñas, a los frutales y avicultura aunque en las grandes posesiones que conservaron su extensión, el uso del suelo no cambió manteniéndose el predominio de los forrajes. Respecto a los sistemas de trabajo, el año 1954 (op.cit.) encontró una correspondencia entre gran propiedad de uso extensivo e inquilinaje tradicional mientras en las propiedades medianas predominaba el inquilinaje modificado o parcial y los jornaleros o asalariados y en las pequeñas propiedades se daba el trabajo familiar y las medierías.

Entre el año 2006 y 2010 el número de trabajadores agropecuarios estables de Calera de Tango disminuyó de 3.835 a 2.864 respectivamente siendo la agricultura la que proporcionaba el mayor número de puestos de trabajo. El empleo temporal agrícola tiene una alta variación anual y por sexo $^{6}$ : Según el Censo Agropecuario del 2007 hubo 2.877 hectáreas plantadas de almendros y uva de mesa en la comuna de Calera de Tango siendo este último el cultivo más intensivo en mano de obra. El número de empresas en la rama agricultura, ganadería, caza y silvicultura con predominio de las empresas agrícolas varió de 186 el año 2006, 174 el 2008 a 159 el 2010. En la actualidad la disminución del empleo agrícola se da en paralelo a la creación de empleos de cuidadores de parcelas, jardineros, empleadas domésticas y en servicios y comercio.

El fundo Rinconada de Lo Ermita se subdividió entre 1940-1941 (Martin, 1960) como parte del proceso de subdivisiones que afectó a los fundos del Valle del Maipo lo que implicó que redujera sus trabajadores. Para 1980 existían veinte y cinco y actualmente solo quedan siete que viven con sus respectivas familias al interior del fundo.

En la década de 1940-50, este fundo de ciento diez hectáreas planas regadas y otras de cerro estaba plantado de viñas y cultivos anuales. Los inquilinos tenían acceso a casa, pequeños trozos de tierra a su alrededor y a leña. Se les remuneraba en salario y se trataba de un tipo de "inquilino en transición" que iba perdiendo las características del inquilinaje tradicional de las grandes haciendas que contaban con más regalías -huerto y talaje- y menos salario. Los hombres se dedicaban al riego, mantenimiento de las viñas y cultivos. En épocas de cosechas llevaban a sus familias, mujeres e hijos, a las vendimias con lo cual engrosaban los ingresos familiares.

En los ochenta y el fundo ya estaba cercado dividiendo las áreas de plantaciones de las casas de los inquilinos cuyos sitios habían disminuido en tamaño dejando apenas la casa y un pequeño patio con salida a la calle. Ya no se podía recolectar leña ni frutas y se comenzó a cobrar la luz que antes era gratuita y lo mismo ocurrió con el agua potable.

Actualmente ha cambiado la organización del trabajo. Los almendrales, cuya producción se procesaba con mano de obra de las familias residentes y gente de afuera, ahora no se cosechan como antes; las almendras se venden con cáscara lo cual ha terminado con el trabajo de mujeres y jóvenes en la temporada de cosecha. De los siete trabajadores agrícolas con residencia en el 
fundo, tres son parientes: el padre y dos de los hijos hombres que tuvieron menos estudios y forman parte de uno de los estudios de caso.

De su lado, la Cordillera de la Costa sufrió la erosión de suelos a lo largo del siglo XX y el bosque nativo fue desapareciendo por la sobre explotación causada por la demanda de madera y leña de la industria, la vivienda y la producción de carbón. A partir de la formulación en 1974 del subsidio a las plantaciones de bosque artificial, el pino insigne y el eucaliptus comenzaron a poblar las zonas cordilleranas de los Andes y la Costa ${ }^{7}$.

El paisaje de bosque nativo, empastadas naturales y de uso agrícola dio paso a la explotación forestal, modificándose así la estructura agraria preexistente de grandes, medianas y pequeñas explotaciones para dar curso a la formación de grandes Sociedades Anónimas de monocultivo forestal. En muchos lugares, el incremento de las superficies de pino y eucaliptus arrinconó al campesinado y comunidades campesinas e indígenas empujándolos a la migración ya que tampoco las actividades silvícolas generan empleo para los lugareños ${ }^{8}$.

Hasta los años ochenta el campesinado que habitó los rincones y pequeños valles costinos se reprodujo gracias a la explotación de sus tierras, la venta de fuerza de trabajo y/o inquilinización en fundos vecinos logrando así preservar la pequeña propiedad como lugar de residencia y de producción para el autoconsumo y el mercado. La expansión del bosque artificial limitó las posibilidades de empleo de los campesinos en las actividades agrícolas puesto que estas desaparecieron ante el avance de las forestales.

Entre las localidades de Cayurranquil y Pilén, al oeste de la comuna de Cauquenes, hubo un notable avance del bosque artificial sobre las tierras de cultivo ${ }^{9}$ desapareciendo predios agrícolas cubiertos por los bosques; paralelamente han aumentado los matorrales en tierras de cultivo lo que podría estar indicando abandono de las actividades agrícolas en el área de pequeña propiedad.

Pilén ${ }^{10}$ se ubica en los faldeos orientales de la Cordillera de la Costa. Se caracteriza por el predominio de la pequeña propiedad. Hacia las tierras de valle existen fundos de tamaño mediano y pequeño que no fueron afectados por la reforma agraria mientras la pendiente es de pequeña propiedad. La ciudad más cercana es Cauquenes (15 km.); hacia la parte alta de la cordillera, llamada La Montaña, se ubica el poblado de Cayurranquil que fue creciendo con las plantaciones forestales desde los años setenta en adelante. En cambio Pilén fue disminuyendo su población fenómeno que se remonta a mediados del siglo $\mathrm{XX}$.

Décadas atrás el mercado matrimonial en la comunidad campesina de Pilén se caracterizaba por la endogamia de las uniones. Las familias eran numerosas, los niños circulaban en la comunidad. Cuando los abuelos quedaban sin ayuda, niños y niñas solían entregarse de una familia a otra, 
siempre entre parientes. Apenas frecuentaban la escuela, niños y niñas a corta edad comenzaban a trabajar ayudando a sus padres y madres. Como resguardo a la vejez, casi siempre una hija mujer permanecía soltera en el lugar para cuidar a sus padres.

A mediados del siglo XX se vivía de la combinación de la pequeña agricultura en tierras propias, de la inquilinización en los fundos vecinos, del trabajo asalariado en los fundos cercanos en época de cosechas y vendimia, de las medierías para cultivos y producción de carbón de campesinos en los fundos de la cordillera ${ }^{11}$, de la recolección de hongos y avellanas en el bosque y de la producción de loza que hacían las mujeres y vendían en la feria y el mercado de Cauquenes. Se recurría al mingaco para las cosechas que los campesinos realizaban en sus propias tierras o en las regalías que obtenían en los fundos donde trabajaban. En las familias con menos tierra, los hombres trabajaban en los fundos como inquilinos o medieros y las mujeres en la alfarería y recolección de frutos del bosque. Hasta los años ochenta hubo esta combinación de distintas formas de trabajo (Valdés, 1990).

El uso del suelo en pequeñas propiedades y fundos se repartía entre viñas, cultivos anuales y hortalizas. Las empastadas artificiales y naturales en los fundos servían a la ganadería. La venta de carbón a pequeña y gran escala daba identidad al lugar junto a la venta de loza de la misma forma que la agricultura, el carboneo y el oficio de locera proveían de identidad a sus habitantes.

En la actualidad, la relación laboral y productiva -mediería, inquilinaje y el mingaco- han sido reemplazadas por el trabajo asalariado con la disminución de efectivos ocupados en la agricultura de manera estable. El trabajo temporal en cambio persiste, incluso incorporando a mujeres que trabajan no sólo en las vendimias sino en la cosecha de arándanos de exportación recientemente plantados.

Las migraciones campo-ciudad, la reconversión productiva de los fundos que han disminuido su fuerza de trabajo y los terremotos que han terminado con las casas de adobe han producido la desocupación del campo lo que va de la mano con la desagrarización de las actividades locales. El avance del bosque artificial desde La Montaña a las tierras de ladera de la Cordillera, el envejecimiento de la población, la venta de tierras de los pequeños propietarios, la disminución de los empleos agrícolas en los fundos y las políticas de subsidio del Ministerio de Vivienda en Cauquenes y sus alrededores han motivado a las generaciones más jóvenes a abandonar el campo.

\section{Familias y grupos de parentesco}

\section{Aldea de Pilén y fundo Rinconada de Lo Ermita}

La diferencia de paisajes, uso del suelo y formas de asentamiento de la población caracterizan los dos lugares en que se ha dado seguimiento a 
las familias y su descendencia. En los inicios de los años ochenta, las parejas que dieron inicio a los dos grupos de parentesco vivían y trabajaban en los fundos La Aldea en Pilén, Cauquenes y Rinconada de Lo Ermita en Calera de Tango (Véase Anexo, Gráficos de parentesco).

Delfina $\left(1936^{12}\right)$, alfarera, hija de campesinos con una exigua cantidad de tierras de Pilén, es la penúltima de seis hermanos. Huérfana de madre a temprana edad fue dejada a cargo de parientes; se casó con su primo Héctor (1932), trabajador agrícola e inquilino de fundo, hijo único de madre soltera de una familia de pequeños propietarios del lugar. De esta unión nacieron doce hijos, la mayoría en la casa con ayuda de una partera del lugar, de los cuales cinco murieron antes del año. De los siete hijos vivos, tres son hombres y cuatro mujeres. De los hermanos/as de Delfina sólo la menor (1924) permaneció en Pilén como ella, los restantes migraron, una hermana (1933) a Santiago a la manufactura textil, otro hermano carabinero vive en Arica. Las migraciones campo-ciudad afectaron a la mayoría de las mujeres del lugar que se ocuparon en Santiago como obreras industriales y empleadas domésticas.

Leontina (1939), fue la décima de doce hijos de una pareja en que su padre era trabajador agrícola en los fundos Águila Sur, Rinconada de Maipú, Viluco todos al sur de Santiago mientras su madre fue ordeñadora en Águila Sur a cambio de casa. Sus hermanos fueron inquilinos y asalariados en estos fundos y sus alrededores y sus hermanas se unieron a inquilinos y asalariados agrícolas. Leontina a su vez formó pareja con Juan (1931) hijo de madre sola, nacido en un fundo de Rapel donde vivía su madre y tíos/as. Juan se desenvolvió como peón rural itinerante; desplazándose por distintos fundos desde joven hasta que obtuvo trabajo estable en el Fundo Lo Ermita donde se instaló con Leontina a la que conoció en Viluco. La pareja tuvo siete hijos, los dos mayores murieron.

Antes del matrimonio Héctor, oriundo de Pilén y Juan originario de Rapel, solían trasladarse de un fundo a otro, de un empleo agrícola a otro. Delfina y Leontina, al igual que otras mujeres de su generación trabajaban desde pequeñas en el dominio doméstico y en la agricultura. Delfina aprendió en la niñez el oficio de locera que desarrolla hasta ahora; Leontina aprendió de su madre a santiguar, componer huesos y a sanar con hierbas lo que transformó en un oficio haciendo hierbas medicinales para la venta. Al formar pareja continuaron trabajando ya sea en la pequeña producción agro-ganadera, como Leontina, también asalariada temporal en viñas y frutales en el fundo o produciendo loza para el mercado como ocurrió con Delfina y una de sus hermanas junto a la mayoría de las mujeres de Pilén que no migraron.

Las dos hijas mayores de Delfina migraron a Santiago tal como lo hizo su hermana y otras mujeres del lugar para trabajar como obreras textiles y empleadas domésticas. Hombres y mujeres de la generación mayor, incluso los hijos mayores de estas dos parejas en su infancia no cursaron más de dos o tres años de educación primaria ya que tuvieron que ayudar a 
sus familias desempeñándose en trabajos agrícolas o ayudando en las tareas domésticas. Un hijo hombre de cada familia sin embargo logró realizar estudios técnicos entre 1970-73.

A comienzos de los años ochenta ${ }^{13}$, la pareja de Delfina y Héctor y la de Juan y Leontina vivían en los fundos La Aldea y Lo Ermita en las comunas de Cauquenes y Calera de Tango, respectivamente. Héctor y Juan trabajaban como inquilinos mientras el resto de la familia, mujeres e hijos, desempeñaban diversas labores asalariadas, de pequeña producción agrícola y alfarería en el caso de Pilén. Dos de los hijos de Héctor y Delfina trabajaban en la agricultura, uno en el fundo La Aldea junto a su padre y otro en un fundo vecino. El hijo mayor que logró hacer estudios técnicos durante la Unidad Popular trabaja hasta ahora como obrero en industrias y construcción de caminos y fijó su residencia en Cauquenes donde vive su familia mientras él trabaja en distintos lugares del país. Las dos hijas menores completaron su educación básica y media a diferencia del resto de los hermanos/as. De los hijos de Juan y Leontina, el mayor (con estudios técnicos) abandonó el fundo y se desempeña hasta ahora como obrero industrial; los otros dos hijos hombres permanecieron como trabajadores agrícolas con casa y trabajo en el fundo donde vive y trabaja hasta ahora el padre. La hija mayor sólo estudio educación básica; la menor completó la educación media, terminó estudios de Técnico Agrícola y luego estudió Pedagogía en Educación Básica en la universidad.

Juan al igual que otros hombres de su generación participó en sindicatos lo que le significó ser víctima de la represión después del golpe de estado. Héctor en cambio, al igual que otros inquilinos de Pilén tuvo una movilidad y participación social reducida a diferencia de su mujer Delfina, quien por su labor como alfarera y sus constantes idas al mercado y ferias tuvo una mayor conexión con el mundo exterior al fundo y a la aldea vinculándose con la Cooperativa Campesina de Cauquenes, además de participar en el Centro de Madres. Por su parte Leontina, también participó en el CEMA y posteriormente, al igual que Delfina fueron parte de la Cooperativa Almacén Campesino que reunía a artesanos de todo el país.

Ambas jugaron un rol importante en el ámbito público y privado, participaron en organizaciones sociales $\mathrm{y}$ en el reparto de funciones y recursos en la familia, asignando tareas a los diferentes hijos, redistribuyendo recursos económicos provenientes del trabajo agrícola asalariado de sus parejas y de ellas, de la venta de sus propios productos (loza y yerbas medicinales) y de los ingresos de algunos hijos o de las remesas enviadas por las hijas que trabajaban en Santiago. Supieron utilizar sus contactos con el mundo exterior (compadres mejor situados socialmente, profesionales, funcionarios públicos, etc.) para lograr beneficios para sus familias.

Los vínculos con el exterior, en los años ochenta en medio de la crisis económica signaron las vidas de Delfina y Leontina permitiendo su participación social al alero de ONGs lo que amplió sus referentes sociales. Los hijos habían crecido, los mayores estaban trabajando, los menores 
estudiando y en la casa permanecía una hija supliendo a la madre en las labores domésticas. Esto permitió que Juan y Héctor establecieran una relación paternal especialmente cercana con las hijas que quedaban a cargo del hogar mientras sus madres salían.

Los hijos/as de ambas parejas tuvieron una escolaridad mayor que sus padres, aunque con diferencias entre hermanos/as lo que se explica porque algunos tuvieron que salir tempranamente a ayudar en las labores agrícolas y domésticas o migraron a la ciudad. Los/as menores en cambio crecieron en un período en que se había incrementado los años de escolaridad obligatoria lo que los favoreció respecto a sus hermanos mayores que tendieron a permanecer en la agricultura aunque dos de ellos, uno de cada familia, lograron acceder a estudios técnicos lo que les posibilitó abandonar el trabajo agrícola.

De la familia de Héctor y Delfina migraron a Santiago dos de las hijas mayores a trabajar como empleadas en casas particulares. La menor de las hermanas una vez completado sus estudios secundarios también migró a Santiago; gracias a su mayor escolaridad logró obtener trabajo de obrera en una fábrica textil y como administrativa; posteriormente, al quedar embarazada regresó a Pilén. Otra de las hijas menores permaneció en Cauquenes, apoyando a su hermana menor mientras estudiaba y luego haciéndose cargo del hogar parental cuando la madre salía a ferias artesanales. Dos de los hijos varones hoy viven en Cauquenes, uno de ellos trabajando como asalariado agrícola y el otro como obrero. El hijo menor, migró a Santiago donde trabaja en una carnicería.

Las hermanas mayores, se casaron en Santiago y siguieron trabajando, una de ellas tiene dos hijos que cursaron estudios universitarios, la otra no tiene hijos. Ninguna de las dos hermanas menores se casó, una de ellas tiene dos hijos que terminaron estudios técnicos (para trabajar en la Aviación en Santiago) y universitarios (Pedagogía en Educación Básica, profesora en escuela de Chiloé) con apoyo económico de su padre (residente en Santiago) y además tiene dos hijos menores que están en el Liceo en Cauquenes con una nueva pareja. Ella trabaja recauchando neumáticos en una vulcanizadora anexa su casa. La menor que trabajó en Santiago, madre soltera, se instaló en Cauquenes en una casa de subsidio a la vivienda que logró con la ayuda de su madre; su hijo realiza estudios técnicos en Mecánica y ella convive con una pareja -obrero en maquinaria pesada- que la apoya económicamente- y se ocupa de acompañar a sus padres en el campo los fines de semana.

Los hijos hombres de Héctor y Delfina, salvo el mayor que hizo estudios técnicos, frecuentaron la escuela entre seis y ocho años al igual que la hija mayor (que migró a Santiago), se casaron y sus hijos varones realizan estudios técnicos mientras las mujeres estudian pedagogía en la universidad, al igual que sus hermanas menores y los hijos de éstas. 
La familia de Leontina y Juan sigue una tendencia similar: los hijos hombres permanecen en la agricultura (salvo un obrero metalúrgico), se casan, tienen menos hijos que sus padres ( 2 a 4 ) mientras las hijas mujeres alcanzan más años de estudios (las menores) e incluso estudios técnicos y universitarios, lo que se generaliza en la tercera generación. Las carreras pedagógicas son las elegidas por ambos sexos, carreras vinculadas al cuidado del cuerpo como Kinesiterapia y Pedagogía en Educación Física y la mecánica, entre las carreras técnicas y universitarias preferidas por los hombres.

De los cinco hijos de Juan y Leontina, dos de los varones trabajan como su padre en el mismo fundo en que nacieron todos los hijos de la pareja. El otro hermano, obrero vive fuera al igual que las hijas. Una de ella se dedica a producir y envasar hierbas medicinales -lo que hizo su madrey comparte la venta de huevos con su marido y es la que se preocupa y ayuda a los padres. La hija menor es Técnico Agrícola y Profesora de Educación Básica en un colegio particular subvencionado de la comuna. Se ha endeudado con el mejoramiento y ampliación de su casa (no quiere vivir en la pobreza de sus padres) por lo que además los fines de semana trabaja como cajera en un restaurante cercano al igual que su hijo para tener para sus estudios. Vive en una casa de subsidio en la comuna vecina de Padre Hurtado donde también han accedido a casa de subsidio dos de sus hermanos (aunque vivan en el fundo) y su hermana. Es madre soltera de un hijo de 17 años que ingresó el 2013 a la Universidad Andrés Bello a estudiar Kinesiterapia.

A diferencia de la familia de Héctor y Delfina, una parte de la cual reside en Santiago, la familia de Juan y Leontina permanece en el mismo lugar o en sus cercanías y dos de sus hijos hombres se desempeñan en las mismas labores que el padre al igual que cuatro de sus nietos que permanecen en el fundo y trabajan en éste o parcelas cercanas. La generación de las hijas e hijos de estas parejas, encarnan otro modelo de familia, con menor cantidad de hijos y con más años de estudios que sus padres y madres. Las hijas menores suelen marcar una ruptura con respecto a los hermanos y hermanas mayores y sus padres en cuanto no constituyen familia conyugal sino tienen hijos solteras. Representan la entrada a nuevos patrones culturales que están bastante más extendidos entre los y las nietos/as de la generación mayor.

\section{Herencias y rupturas entre generaciones}

Las hijas menores de trabajadores de fundos de ambos lugares conforman una generación de tránsito entre una situación marcada por el analfabetismo o pocos años de estudio de sus padres e incluso de sus hermanos mayores a otra marcada por nuevas expectativas educacionales y laborales; tránsito de familias numerosas a menos hijos y cambio generacional que marca el abandono de la agricultura con el fin de acceder a otro tipo de empleo y de residencia. Ellas y sus hermanos y hermanas han accedido a 
viviendas subsidiadas por el Estado en un medio rural que ha dado lugar a procesos de urbanización en terrenos que hace pocas décadas eran agrícolas. La urbanización de la residencia impulsada por políticas públicas acompaña al proceso de "desagrarización” del empleo, particularmente de las mujeres incluyendo en la generación más joven a ambos sexos. A diferencia de sus madres no se perfilan bajo una identidad definida por la maternidad usualmente acompañada en la generación mayor por el despliegue de oficios que reposan en la cultura campesina y empleos agrícolas temporales que se decodificaron como "ayuda al marido". Más que la maternidad como mandato de género y marca identitaria, lo que aparece en aquellas que bordean los cuarenta años es un lugar en la parentalidad signado por la preocupación de llevar a buen puerto el proyecto educativo de sus hijos, a veces el hijo único, lugar que suele darse en ausencia de los progenitores hombres que sin embargo suelen contribuir económicamente. Esta orientación al logro de la educación se ha incorporado plenamente como aspiración de un tipo de familia que persigue lograr movilidad social por esta vía bajo el lema compartido entre sus padres "para que no sean como uno".

No obstante, esta tendencia convive con otra: familias conyugales, con tres a cuatro hijos en los hermanos y hermanas mayores, el sello de dueñas de casas ocupadas de la familia independientemente que el proyecto educativo esté muy presente en la generación intermedia y las madres se consagren al logro de la educación de sus hijos. Llama la atención entre las hijas mujeres menores el caso de una hija de Delfina y Héctor que cuenta con dos parejas, dos hijos con educación técnica y universitaria (los otros dos en la educación media) y ella desempeñándose en un oficio masculino como la vulcanización de neumáticos en un taller propio (con su pareja) en Cauquenes; a veces "locea” como su madre y es la única que heredó el oficio materno.

Si la migración campo-ciudad entre los hombres y las mujeres de la generación mayor y la intermedia los llevó a emplearse en las ciudades como obreras en la manufactura, empleadas de casa particular, obreros industriales y en la construcción, hoy se hace visible un cambio marcado por estudios universitarios y técnicos sin que necesariamente haya migración a la ciudad o la haya a ciudades cercanas como Cauquenes. Uno de los nietos de Juan, profesor de inglés en un importante Liceo de Hombres de Santiago (con una pasantía en Estados Unidos), que arrendaba un departamento en Santiago, al decidir realizar estudios de Maestría en Linguística volvió a la casa paterna en el fundo para poder ahorrar y pagarla. El hijo de Marta, y nieto de Juan y Leontina que estudia Kinesiterapia vive con su abuelo en el fundo y viaja cotidianamente a la universidad. La mayor escolaridad y cercanía con lo urbano de los más jóvenes se evidencia en el consumo cultural, vestimenta y uso de tecnología, que va desde los teléfonos celulares, pasando por el computador conectado al internet. Para ellos el campo es un lugar para visitar a sus abuelos, incluso para vivir pero trabajando o estudiando en la ciudad. Así como la generación de sus padres vivió el tránsito a la desagrarización (con excepción de los hombres con pocos estudios), estos jóvenes muestran en sus modos de ser y pro- 
yectos de vida que son la generación de la desruralización (Pilén/ Cauquenes). No obstante, la cercanía a la ciudad que ofrece servicios, empleo y lugares de estudios superiores y donde además los medios de transporte se han hecho expeditos permiten vivir en el medio rural y trabajar o estudiar en la ciudad, frenando la salida del campo. (fundo Lo Ermita/Santiago).

Para finalizar: Héctor está jubilado y permanece en la casa propia que compraron en Pilén con un pequeño sitio para huerta que él ayuda a mantener; la casa cuenta con agua, luz y electrodomésticos. Delfina sigue trabajando en la alfarería saliendo a mercados y ferias; los fines de semana son visitados por su hija menor y en las vacaciones de verano reciben la visita de la familia que vive en Santiago. Juan también está jubilado pero trabaja y sigue viviendo en el fundo Lo Ermita en la casa en que nacieron sus hijos, al igual que los hijos que trabajan en el fundo y habitan casas cercanas a la del padre y cuatro de sus nietos hombres. Jubilado, continúa trabajando por el salario mínimo y comparte la vivienda con uno de sus nietos que estudia en la universidad. Leontina vive en la casa que obtuvo a través del subsidio a la vivienda y visita a su marido los fines de semana o bien él la visita a ella. Los hijos hombres de estas dos parejas sin estudios secundarios, técnicos o universitarios permanecen como sus padres trabajando en la agricultura. Los hombres que no son trabajadores agrícolas tienen estudios técnicos logrados en el período de reforma agraria y trabajan como obreros calificados; las mujeres que migraron en cambio, sin estudios, se desempeñaron como obreras en la manufactura pero sobre todo como empleadas domésticas. Independientemente de la educación de los padres la mayoría de los nietos tienen estudios secundarios completos, estudios técnicos y algunos, universitarios.

\section{Conclusiones}

En territorios diferentes -ricas tierras de riego en el Valle del Maipo y pobres tierras de secano en Pilén- donde en ambos lugares son gravitantes las actividades agrícolas, -uno invadido por el negocio inmobiliario y las políticas públicas de vivienda social, otro cercado por el avance del bosque artificial- ocurren fenómenos diferentes pero las tendencias a la desagrarización y desruralización son comunes a ambos lugares y se encarnan en la generación joven que ha aumentado sus años de escolaridad con respecto de sus abuelos, padres e incluso hermanos/as mayores. Sin embargo la urbanización de los espacios rurales junto a la cercanía de la ciudad frenan la expulsión a la ciudad ya que la residencia rural es compatible con el empleo no agrícola y los estudios. Los "espacios del capital" habitados por el bosque artificial, en un caso han producido despoblamiento y migración a una ciudad cercana (Cauquenes); en otro, donde la ciudad ha avanzado se ha producido una disminución de los empleos agrícolas (fundo Lo Ermita) y retención de la cohorte más joven en zonas rurales urbanizadas pues los estudios o el trabajo son accesibles aun viviendo en el campo. 
En el pasado las vidas de quienes habitaban el campo así como las identidades de hombres y mujeres se circunscribían al lugar que hacía referencia al espacio físico, dónde se nacía, el tipo de actividades laborales, domésticas u oficios que se realizaban, dimensiones determinantes en la identificación de cada cual con respecto a otros: los del pueblo, de otros fundos o comunidades cercanas, los hombres, las mujeres.

Los contactos con la urbe de hombres y mujeres que abandonaron el campo incidieron en cambios en las formas de vida rurales de los que se quedaron visibles en el manejo de los asuntos domésticos de las mujeres (De Certeau, 1990) y la crianza de los hijos; y en los hombres en el conocimiento y manejo de maquinaria y herramientas y, en particular, en el plano organizativo y político. El abandono del campo era por la falta de empleo y bajas remuneraciones, la sujeción a patrones, administradores de fundos, restricciones económicas de las familias pero también a las reglas que imponía la familia toda vez que se trabajaba gratis al alero del padre o de la madre.

Para quienes permanecieron en el campo, el patrón, el mercado, el padre o el marido, la parentela, definían el universo de las relaciones sociales de hombres y mujeres, adolescentes y niños. Instituciones como la iglesia, la escuela, el compadrazgo, fiestas religiosas, hechos vinculados a las edades de la vida como nacimientos, bautizos, matrimonios, defunciones convocaban a los miembros de la comunidad campesina a encuentros esporádicos.

Las identidades de género estaban fuertemente ancladas en el trabajo (hombres) y la familia (mujeres) aunque en la generación mayor el trabajo y ciertos oficios eran gravitantes en la afirmación de las identidades femeninas en la comunidad campesina (como loceras y santiguadoras/sanadoras).

La reforma agraria rompió con el aislamiento de las poblaciones rurales por la emergencia del sindicalismo y la cercanía de los campesinos con las instituciones del Estado. Para las mujeres este aislamiento disminuyó con el acceso a las escuelas de sus hijos, a los hospitales (Rebolledo, 1991) y a los espacios organizativos propiciados por el Estado para su mejor desempeño como madres. La generación intermedia estuvo expuesta a la emergencia de la educación como horizonte, a la salida de algunos hombres de los empleos agrícolas gracias a estudios técnicos y aparece como atributo de la identidad femenina el trabajo junto a una profesión. La identidad de las mujeres centrada en la familia se debilita emergiendo el proyecto personal como un nuevo componente de los cambios que ha experimentado la sociedad. Sin embargo en esta generación se hacen visibles dos tendencias: la descrita que marca procesos de individuación y una opuesta donde se reproduce una identidad centrada en la familia y la maternidad extendiéndose el lugar de las mujeres no sólo a la familia propia sino a la de sus padres por el cuidado que reclaman mientras la madre está en lo público. Las mujeres salen del campo y de las actividades agrícolas mientras algunos hombres de esta generación se mantienen en la agricultura. 
Para las generaciones jóvenes los referentes se han ampliado en concordancia con los cambios que ha experimentado la sociedad. Los lugares relativamente cerrados y aislados que albergaron a comunidades campesinas y a los trabajadores de fundos con formas de vida volcadas sobre sí mismas dejaron de existir; las antiguas fronteras entre campo y ciudad se disolvieron (a partir de la reforma agraria) y las vidas actuales se despliegan fuera del tiempo circular de las labores culturales conducidas por las estaciones. El tiempo y el espacio se rehízo con la apertura de caminos, el acceso a los medios de comunicación, telefonía celular, internet y transporte.

Entre los jóvenes la noción de lugar se ha desprendido de la naturaleza ampliándose no sólo a la ciudad sino a otros países en su misma experiencia formativa y en sus expectativas de profesionalización.

Son manifiestas las diferencias en las maneras de pensar, hacer, habitar, situarse en el mundo, identificarse entre las generaciones mayores y los más jóvenes. De la misma manera que la sociedad se transformó cambiaron las expectativas de las personas y emergió un nuevo sujeto anteriormente opacado por la familia y la comunidad campesina. Como señala Touraine (1996: 65): “el sujeto personal solo puede formarse apartándose de las comunidades demasiado concretas, demasiado holistas, que imponen una identidad fundada en los deberes más que sobre los derechos, sobre la pertenencia y no sobre la libertad”. Aún inscritas en estos vectores, las trasformaciones identitarias se manifiestan en la pérdida de importancia de las normas familiares que modelaron el principio de autoridad centrado en el padre y/o esposo, a la vez que cambió el concepto de honor frente a la disminución en importancia del matrimonio y al aumento de la filiación no matrimonial.

Hombres y mujeres no han vivido de igual forma estos procesos inscritos en la modernización y urbanización de las formas de vida rurales. Marcadas diferencias por sexo existen entre la generación mayor e intermedia tendiendo a mayores similitudes entre ambos sexos en las aspiraciones de la generación más joven dotada de acceso a tecnología en comunicación y con mayor escolaridad.

Uno de los hallazgos de este estudio es que pese a las diferencias entre lugares parte de territorios más amplios, impacto de la vida urbana, calidad de las tierras, etc., la joven generación de ambos lugares sigue las mismas orientaciones: desagrarización, urbanización y aumento de la escolaridad, aunque se mantengan los contactos con el medio rural e incluso se viva en el campo. Siendo la educación el factor más gravitante en estas transformaciones sociales, se hace visible en ellas el impacto que tuvo el Estado en el largo plazo a través de las reformas educativas de los años sesenta y comienzos de los setenta y el posterior acceso a establecimientos de educación media y superior privados con el concurso de las solidaridades de la familia y la red de parientes para solventar la permanencia de los nietos y nietas de los inquilinos en el sistema educativo. 
Independientemente de la localización de las familias (zonas de secano aislada, zona de riego urbanizada), los procesos de cambio y de movilidad social son semejantes lo que habla de la incidencia de las políticas públicas y del mercado de bienes materiales y simbólicos en la generalidad de las trayectorias de los menores de cuarenta años y particularmente en los más jóvenes.

La hibridación rural-urbano se manifiesta en el empleo (jóvenes rurales y urbanos trabajan en la temporada para ayudarse en los estudios) así como en el transporte que permite ir y venir entre campo y ciudad en tiempos cortos, uso de celulares e internet y consumo cultural y electrodomésticos que asemejan campo y ciudad. Es importante señalar que pese a la tecnificación de las tareas domésticas, el acceso a lavadora, refrigerador, etc., estas actividades siguen en manos de las mujeres en particular en la generación mayor e intermedia aunque en las actividades públicas haya cambios notables lo que se hace visible en las mujeres mayores y en menor medida en la generación intermedia que ha permanecido en el campo (hijas y nueras).

Los impactos de las políticas públicas se hacen visibles en el acceso a vivienda que suele lograrse cuando las mujeres movilizan recursos económicos para acceder a ellas (el ahorro previo). Con respecto a la educación, es la familia la que provee para sacar adelante a los jóvenes en los estudios secundarios, técnicos y universitarios. El papel de la "economía oculta del parentesco", referido a las estrategias y movilización de recursos que operan fuera de las instituciones de la sociedad y dentro de la familia y la red de parentesco (Goody, 1986:249-263) es clave para comprender estos procesos.

El proceso de individuación de los hijos, la posibilidad de trabajar y estudiar, han cambiado las identidades e intereses de los jóvenes que han podido liberarse de la tutela de los padres aunque en ningún caso de su apoyo para salir del campo y del trabajo en la agricultura. No obstante, este salto adelante -de hombres y mujeres- es solo posible con el concurso de las mujeres de la familia que ahorran y transfieren recursos para que ellos estudien y saquen "los pies del barro" aunque las mujeres no saquen las manos de la cocina. El lugar de ellas en la redistribución de bienes y recursos dentro de la parentela permite favorecer a las hijas mujeres, más aún cuando no están casadas o no tienen pareja, y a las nietas y nietos.

Son las familias las que a través de la movilización de recursos y capitales económicos y simbólicos las que operan como factores de movilidad social de las hijas menores y de los jóvenes de la generación menor permitiéndoles beneficiarse de las políticas públicas y llevar adelante las aspiraciones educativas que tienen un alto costo. Sin embargo, esta "economía oculta del parentesco" opera en un doble sentido permitiendo que algunas hijas den este salto adelante, y otras permanezcan en la casa atendiendo no solo su propio hogar sino el de sus madres que buscan también legitimidad en el espacio público. 


\section{Notas}

${ }^{1}$ Proyecto Fondecyt 1120425 "Cambio en las identidades y relaciones de género en el ámbito rural de Chile Central”. Investigadora responsable Ximena Valdés, ximena.valdes@cedem.cl; coinvestigadora Loreto Rebolledo, mareboll@u.uchile.cl.

${ }^{2}$ El recuerdo personal, cargado de sentido para quien lo conserva, pese a su carga de subjetividad y a la reinterpretación de que es objeto desde el presente del sujeto que lo habla, constituye una fuente oral fundamental, pues se basa en experiencias propias de quien lo porta, experiencias que muchas veces solo se traspasan de manera informal y abreviada como anécdotas privadas de familia o de grupos afines pero además es parte de un contexto mayor (cfr. Prins, 1993).

${ }^{3}$ Las genealogías son la expresión gráfica de los resultados de la información sobre una o más familias que incorpora a ascendientes y descendientes recolectada a través de entrevistas y procesada y analizada. (Davinson, 2006)

4 A inicios del gobierno militar, se formuló la Política de Desarrollo Agrario y Rural (ODEPA, 1974) que favoreció la desocupación de población en las propiedades agrícolas y estableció nuevas formas de asentamiento de la población en "villorrios rurales” donde se asentó a los expulsados de la agricultura producto de la contra reforma agraria. Desde 1990 las políticas públicas de vivienda han ocupado tierras agrícolas en las márgenes urbanas.

${ }^{5}$ En el estudio de Martin de 1960 se consignaron 49 propiedades en 1896, de éstas, tres menores de 100 hectáreas. En 1954 eran 330 de las cuales un 86 por ciento eran menores de 100 hectáreas esto muestra la temprana subdivisión que afectó a estos territorios del Valle del Maipo.

${ }^{6}$ Según el Censo Agropecuario en la temporada agrícola 2006-2007en el $1^{\circ}$ trimestre trabajaron 302 hombres y 191 mujeres, en el $2^{\circ}$ trimestre, 385 hombres y 324 mujeres, en el $3^{\circ}$, 833 hombres y 647 mujeres, y en el $4^{\circ}$ trimestre 801 hombres y 699 mujeres lo que se explica por el uso del suelo en la comuna.

${ }^{7}$ Esto incrementó la superficie forestal del país -según los Censos agropecuarios, de 74\% entre 1976 y 1997 y del 35\% entre 1976 y 2007-, estableciéndose amplias superficies de monocultivo de bosques y el encadenamiento de la silvicultura con la industria de la madera y celulosa nacional e internacional.

${ }^{8}$ El trabajo temporal en las forestales la tala y procesamiento de la madera recae en trabajadores subcontratados de áreas urbanas. Los nuevos arreglos espaciales provocados por el crecimiento del bosque producen vaciamiento de antiguos establecimientos humanos y el crecimiento de lugares débilmente poblados como consecuencia de la instalación de campamentos (Santana, 2006).

${ }^{9}$ Fotointerpretación fotografía aérea de La Aldea 1979 y 1994 e imágenes satelitales 2010. Cambios en la cobertura vegetacional y configuración predial en la localidad de La Aldea realizada por Alejandro Gutiérrez, enero 2014.

${ }^{10}$ La información de Pilén y Cayurranquil proviene del trabajo de campo realizado en los años 1986, 2001, 2012 y 2013. 
Polis, Revista Latinoamericana, Volumen 14, $N^{\circ}$ 42, 2015

${ }^{11}$ La mediería en carbón fue el mecanismo usado por los propietarios de fundos de La Montaña para desbrozar sus campos de bosques y dedicar ciertas áreas a cultivos y ganadería.

${ }^{12}$ Los números entre paréntesis señalan el año de nacimiento.

${ }^{13}$ Cuando se hicieron las primeras entrevistas. 


\section{Bibliografía}

Alfonso, A., Gómez, S., Klein, E., Ramírez, P. (1970), Movimiento campesino chileno, 2 tomos, ICIRA, Santiago.

Bauer, A (1994), La sociedad rural chilena: desde la Conquista española hasta nuestros días. Ed. Andrés Bello, Santiago.

Bengoa, J. (2013), "Rural Chile Transformed : Lights and Shadows”. Journal of Agrarian Change, pp. 466-487, Wiley Blackwell, Oxford, U.K.

Bengoa, J. (2012), Valle Central. Memorias, patrimonio y terremoto en haciendas y pueblos de Chile Central. Catalonia, Santiago.

Bengoa, J. (1988), El poder y la subordinación. Historia social de la agricultura chilena, Tomo I, SUR, Santiago.

Canales, A y Canales, M.(2013), “De la metropolización a la agropolis. El nuevo poblamiento urbano en Chile actual”. Revista Polis 34, Santiago.

Caro, P. (2012), “Caso de Chile”. En: Empleo y condiciones de trabajo de mujeres temporeras agrícolas. CEPAL/FAO/OIT, Santiago. Pp. 143-221.

Davinson, G. (2006), Herramientas de investigación social. Guía práctica del Método Genealógico. Universidad de La Frontera, Temuco/Universidad Iberoamericana, México.

De Certeau, M. (1990), L'invention du quotidien. L'art de faire, Folio/ Essais, Gallimard, Paris.

De Gaulejac, V. (1999), L'histoire en héritage. Roman familial et trayectoire sociales. Ed. Desclée de Brouwer, Paris.

De Gramomont, H. (2009), “La desagrarización del campo mexicano”. En: Convergencia. Revista de Ciencias Sociales, num 50. Pp. 15-55, México.

Dirven, M. (2004), “El empleo rural no agrícola y la diversidad rural en América Latina”. Revista de la CEPAL 83, Santiago. Pp. 49-69.

Gómez, S. (2014), "La tenencia de la tierra y sus problemas en América Latina y el Caribe”. En: Revista ALASRU. Conferencias magistrales y mesas redondas. $10^{\circ}$ Congreso sociedades rurales latinoamericanas, diversidades, contrastes, alternativas. № 10, México. Pp. 147-168.

Domíngues, J. M. (2002), “Generacoes, modernidade e subjetividade”. Revista Social USP, S. Paulo, 14 (1). Pp. 67-89.

Gómez, S. y Echenique, J. (1988), La agricultura chilena. Las dos caras de la modernización, FLACSO/AGRARIA, Santiago. 
Goody, J. (1986), “La evolución de la familia y el matrimonio en Europa”, Editorial Herder, Barcelona.

Guber, E.G. y Lincoln Y. (1981), Effective evalutation: improving de usefulness of evaluation results through responsive and naturalistic approaches, Jossey Bass, San Francisco.

Gunderman, Hans (2004), "El método de estudio de casos”. En: Observar, Escuchar y Comprender. Sobre la tradición cualitativa en investigación social. M.L. Tarrés (coord.). FLACSO/El Colegio de México, México.

Harris, C.C. (1986), Familia y sociedad industrial, Ed. Península, Homo Sociologicus 37, Barcelona.

Héritier, F. (1996), Masculin/féminin. La pensée de la différence. Tome 1. Odile Jacob, Paris.Héritier, F. (1981) L'exercice de la parenté. Hautes Etudes, Gallimard, Seui, Paris.

Harvey, D (2007), Espacios del capital. Hacia una geografía crítica, AKA, Madrid.

INE. Censo Agrícola Ganadero 2007 y 1997, Santiago.

Kay, C. (2009), “Estudios rurales en América Latina en el período de globalización neoliberal. ¿Una nueva ruralidad?” Revista Mexicana de Sociología, Vol. 71, N4. Pp. 607-645.

Lara, S.(2010), “Movilidad y migración de familias jornaleras: una mirada a través de genealogías”, en Revista Empiria $N^{o} 19$, enero-junio 2010, pp. 183-203. ISSN: 1139-5737.

Lamas, M. (2013), El género. La construcción social de la diferencia sexual. Ed. Miguel Angel Porrúa, México.

Montecino, S., Rebolledo, L. Willson, A. (1993), Diagnóstico sobre inserción laboral de mujeres mapuche rurales y urbanas.PIEG, Universidad de Chile/SERNAM, Santiago.

Martin, G.E (1960), La división de la tierra en Chile Central. Facultad de Filosofía y Educación, Departamento de Geografía, Instituto de Geografía. Editorial Nascimiento, Santiago.

Ortega, E. (1987), Transformaciones agrarias y campesinado. De la participación a la exclusión. CIEPLAN, Santiago.

Osandon, L. y González, F. (2014), “La educación de masas durante la Unidad Popular: una nueva escuela para toda la comunidad”. En: Fiesta y drama. Nuevas historias de la Unidad Popular, Julio Pinto (ed.), LOM, Santiago. 
PNUD (2008), Desarrollo Humano en Chile Rural. Seis millones por nuevos caminos, PNUD, Santiago.

Prins,G.(1993), “Historia Oral” en Formas de hacer historia, Peter Burke, editor,Alianza Editorial, Madrid.

Rebolledo, L (1991), Fragmentos. Oficios y percepciones de las mujeres del campo, CEDEM, Santiago.

Santana, R. (2006), Agricultura chilena en el siglo XX: contextos, actores y espacios agrícolas, CEDER/Centro de Investigaciones Diego Barros Arana, Colección Sociedad y Cultura, Santiago.

Tarrés, M.L. (2004) (coordinadora), Prólogo de Observar, escuchar y comprender.Sobre la tradición cualitativa en la Investigaación social.FLACSO-México-El Colegio de México

Testart, A.(2014), L'amazone et la cuisiniere. Anthropologie de la división sexuelle du travail. Gallimard, Paris.

Tinsman, Heidi (2009), La tierra para el que la trabaja. Género, sexualidad y movimientos campesinos en la Reforma Agraria chilena, LOM, Santiago.

Touraine, A. (1997), ¿Podremos vivir juntos? La discusión pendiente: El destino del hombre en la aldea global, Fondo de Cultura Economico, Argentina.

Valdés, X., Rebolledo, L., Pavez, J., Hernández, G.(2014), Trabajos y familias en el neoliberalismo. Hombres y mujeres en las faenas de la uva, el salmón y el cobre. LOM, Santiago.

Valdés, X (2012), “Desincronización temporal y espacial entre trabajo y familia: Hacerse el salario en las migraciones estacionales de los/as temporeros/as de la uva”. Revista Polis 31.

Valdés, X. (1988), “La feminización del mercado de trabajo agrícola en Chile Central”, En: Mundo de Mujer. Continuidad y cambio, CEM, Santiago.

Recibido: 12.05.2014

Aceptado: 18.03.2015 